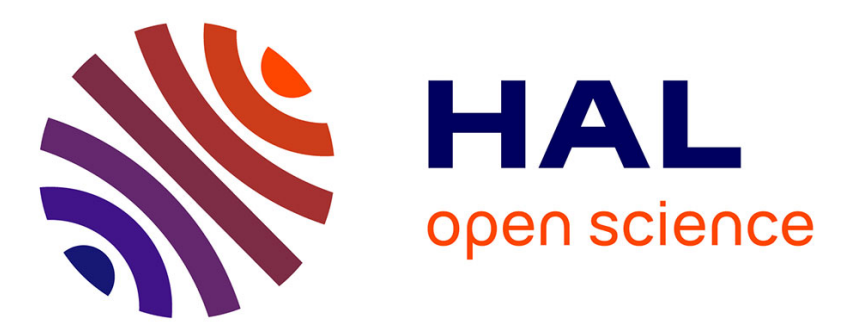

\title{
Representing and reasoning about different viewpoints: an agronomy application
}

Madalina Croitoru, Rallou Thomopoulos

\section{To cite this version:}

Madalina Croitoru, Rallou Thomopoulos. Representing and reasoning about different viewpoints: an agronomy application. ICCS: International Conference on Conceptual Structures, Jul 2009, Moscow, Russia. pp.128-140, 10.1007/978-3-642-03079-6_10 . lirmm-00410637

\section{HAL Id: lirmm-00410637 https://hal-lirmm.ccsd.cnrs.fr/lirmm-00410637}

Submitted on 21 Aug 2009

HAL is a multi-disciplinary open access archive for the deposit and dissemination of scientific research documents, whether they are published or not. The documents may come from teaching and research institutions in France or abroad, or from public or private research centers.
L'archive ouverte pluridisciplinaire HAL, est destinée au dépôt et à la diffusion de documents scientifiques de niveau recherche, publiés ou non, émanant des établissements d'enseignement et de recherche français ou étrangers, des laboratoires publics ou privés. 


\title{
Representing and reasoning about different viewpoints: an agronomy application
}

\author{
Madalina Croitoru ${ }^{1}$ and Rallou Thomopoulos ${ }^{2,1}$ \\ 1 LIRMM (CNRS and Univ. Montpellier II), F-34392 Montpellier cedex 5, France \\ croitoru@lirmm.fr \\ 2 INRA, UMR1208, F-34060 Montpellier cedex 1, France \\ rallou.thomopoulos@supagro.inra.fr
}

\begin{abstract}
Real-world applications are often complex systems where several ways of analysing a given situation can be expressed, depending on actors' viewpoints. This paper proposes a semantically sound syntactic extension to Conceptual Graphs, namely Conceptual Graph Assemblies (CGAs), that allows the representation of multiple viewpoints on the same situation. Several reasoning mechanisms, based on the projection operation, corresponding to different strength levels and adapted to multi-viewpoints situations are then demonstrated. Several modelling scenarios are then proposed and our work is put in the context of real world examples from the agri-food domain.
\end{abstract}

\section{Introduction}

Quality control within agri-food chains relies on numerous criteria: nutritional, functional, sanitary, environmental, economical, etc. The management of food quality has to reconcile several facets constituted by these criteria. Moreover, the objectives of quality are based on several actors: technicians, managers, users, scientists, professional associations, public communities, etc. The importance attached to the different quality criteria varies according to the considered actors. These elements lead to the following open research questions: "how to represent, within a knowledge representation model, these contradictory viewpoints?"; "how to take into account, by the reasoning mechanisms, the interests of the different involved actors?"

The current structure of chains is questioned as for system perenniality, protection of the environment, public health issues, cost and energy. The actors' viewpoints are divergent, hence it is necessary to define representational and reasoning mechanisms able to model and take into account the balance between viewpoints, and the risks and benefits they imply. Our general objective is the conception of a decision support tool for the actors of an agri-food chain, in presence of contradictory viewpoints and priorities.

In this context, as a first step, we built a knowledge-based system able to represent the different kinds of knowledge needed, initially provided with consistency checking, querying and symbolic simulation mechanisms. Given that the information sources are both experimental data extracted from the domain 
literature and expert statements, the intuitiveness and proximity to natural language of the representation language are essential features. Moreover, the experts should be able to understand the reasoning on their modelling and to validate it, thus reasoning should be done directly on the knowledge representation and feedback intuitive. Finally, a logical semantics is desirable as a foundation for reasoning and the language should be flexible enough to be easily extended to new features. For these reasons, conceptual graphs were initially chosen as the knowledge representation and reasoning language for this specific application.

However, conceptual graphs cannot easily represent different, potentially contradictory viewpoints, and moreover, rigorous mechanisms for reasoning about this type of knowledge have not been put into place. In this paper we present a formalism that allows the representation of such contradictory, inconsistent type of knowledge for this application along with sound and complete syntactic operation for manipulation.

A simple case of this problem has been addressed by Puder [9] who considered alternative descriptions for one concept. He built a tree with this concept as a root node, and used this structure for service trading in the AI-Trader project ${ }^{3}$. This work is not sufficient in the context of the agronomy domain where whole sentences could be debated and argued upon. In [10], an approach for viewpoint representation is proposed in the framework of the conceptual graph model, however it concerns the expression of facets of concepts in an ontology, i.e. the terminological part of the model (the support), and does not treat the commensal representation of several viewpoints in the assertional knowledge. Another approach for representing viewpoints in the conceptual graph model is based on nested graphs. They have been introduced at a descriptive level by Sowa [12] as a way of representing contexts by structuring knowledge by levels, and studied in further works such as $[7,8]$. Typed nestings were introduced by [2], which allows to specify the relationship (description, explanation, etc.) between the surrounding vertex and one of its descriptions and thus to explicitly attach several descriptions to the same vertex. Each description can then be viewed as a viewpoint, as proposed in [13] which more specifically focuses on how to associate specific vocabularies with contexts. A drawback of the nested graph approach is that it does not allow inter-viewpoint reasoning, such as inter-viewpoint projection or detection of contradictions between viewpoints. In $[5,6]$ an extension to Conceptual Graphs was proposed to further address the above mentioned modelling needs. However, the proposed formalism was lacking in rigorousness by the fact that the combinatorial structures proposed were not complete with respect to the proposed semantics [5]. While this problem has been partially solved in [6] the lack of a concrete practical framework to address the concrete modelling needs of the agri-domain was still to be addressed.

In this paper we extend this formalism by showing different combinatorial structures of defining sound and complete viewpoints as well as demonstrating their applicability for the above mentioned problem in the agronomy domain. Section 2 presents a motivating example, Section 3 introduces the formalism,

\footnotetext{
${ }^{3}$ http://www.puder.org/aitrader/
} 
Section 4 shows how CGAs can be used in conceptual modelling, finally Section 5 concludes with some perspectives.

\section{Motivating example}

Conceptual graphs [12,11] (CGs) are a logical, graph-based approach to knowledge representation that introduce a clear distinction between ontological and asserted knowledge. More specifically, a Conceptual Graph represents knowledge as a support and an associated bipartite graph. The support encodes the ontological, background information. It consists of a concept and a relation taxonomy along with the markers used to denote instances or generic concepts. The factual information is depicted as a bipartite graph where one partition class, the concepts, is represented using square nodes, and the other, the relations, is represented using ovals. An example of a Conceptual Graph is depicted in the figure below:

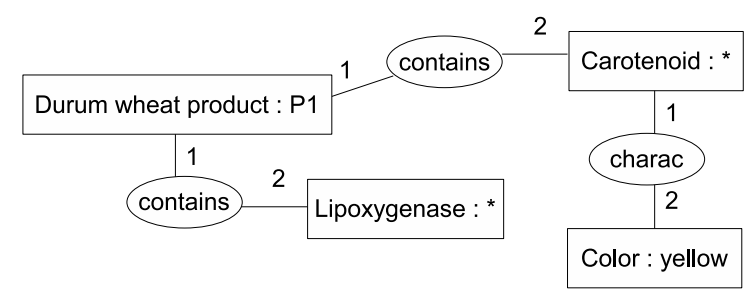

Figure 1. Example of a Conceptual Graph

The conceptual graph in this figure states that the durum wheat product $\mathrm{P} 1$ contains a lipoxygenase and carotenoid which is characterised by the yellow color.

Reasoning with Conceptual Graphs means translating the Conceptual Graph into FOL (First Order Logic) formulae and employing FOL deduction. Another method looks at finding a homomorphism (projection) between two graphs defined on the same support. These two methods have been proven equivalent $[12,1]$.

However, Conceptual Graphs can only represent static, "snapshot" facts about the world. Indeed, the support encodes the hierarchies which classify the entities and relations we need to describe a certain scene, while the bipartite graph represents that scene. We do not have a proper built-in mechanism to describe alternative scenes (e.g. as viewed from different/inconsistent view points). An example of the expressivity needed for this application is depicted in figure 2 .

In this figure two viewpoints are represented about the information given by a conceptual graph: the scientist viewpoint (denoted "Sc.") and the marketing viewpoint (denoted "Mk."). The scientist view indicates that the durum wheat product P1 contains carotenoid characterised by the yellow color, lipoxygenase 


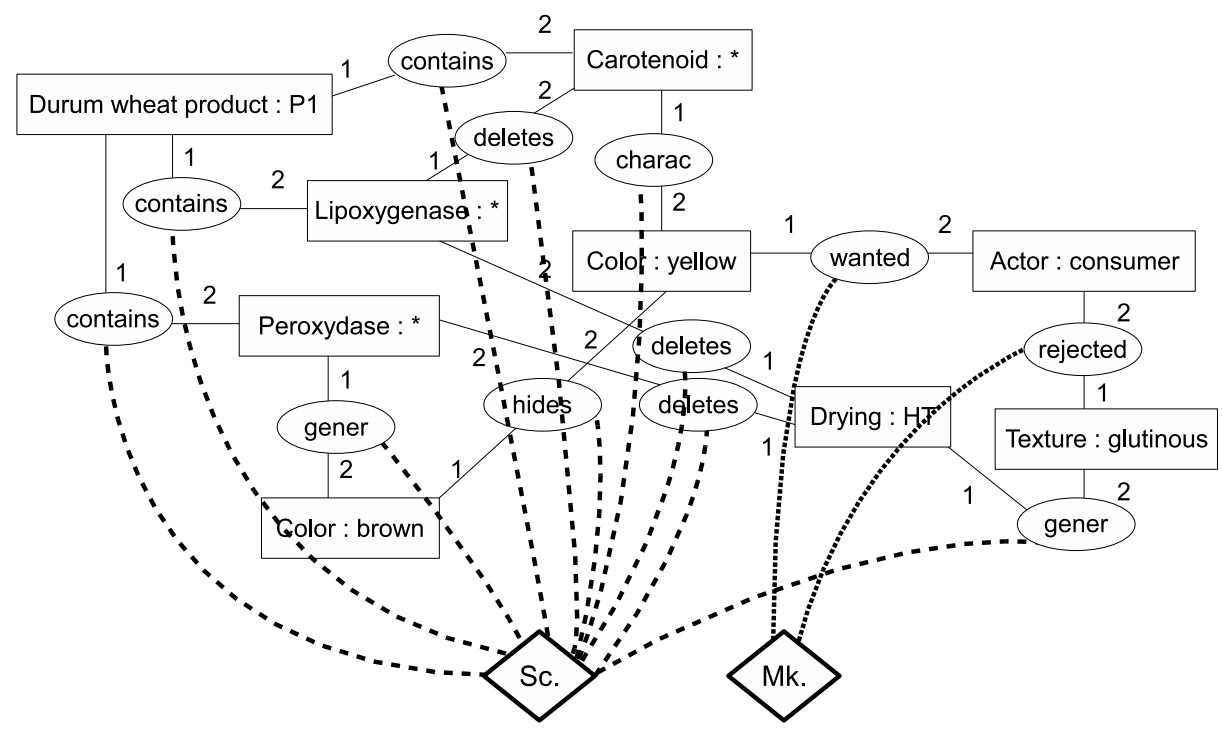

Figure 2. Multiple viewpoints in the agri-food application

that deletes carotenoid, and peroxydase that generates a brown color which hides the yellow one. It also indicates that the HT (High Temperature) drying deletes lipoxygenase and peroxydase and generates a glutinous texture. The marketing view indicates that the yellow color is wanted by the consumer and that the glutinous texture is rejected by the consumer.

In this paper we propose a syntactic, semantically sound mechanism for representing the expressivity needs mentioned above. Viewpoints are represented using different combinatorial grouping in the Conceptual Graphs Assemblies (CGA). Reasoning about viewpoints is done using an extension of the projection mechanism that respects the combinatorial structure induced by the CGA. In the following example the query is searched in all of the viewpoints therefore denoting a consensus.

For example, consider the following simple query composed of a single concept vertex:

Property : *

Figure 3. Example of a simple query

The meaning of searching for this query in all of the viewpoints is highlighting product properties that are of interest for all of them, e.g. in figure 2 for both scientists and marketing. In the example of figure 2, there are two answers to 
this query that appear in all the viewpoints. These answers correspond to the concept vertices represented in greyed out shade in figure 4 .

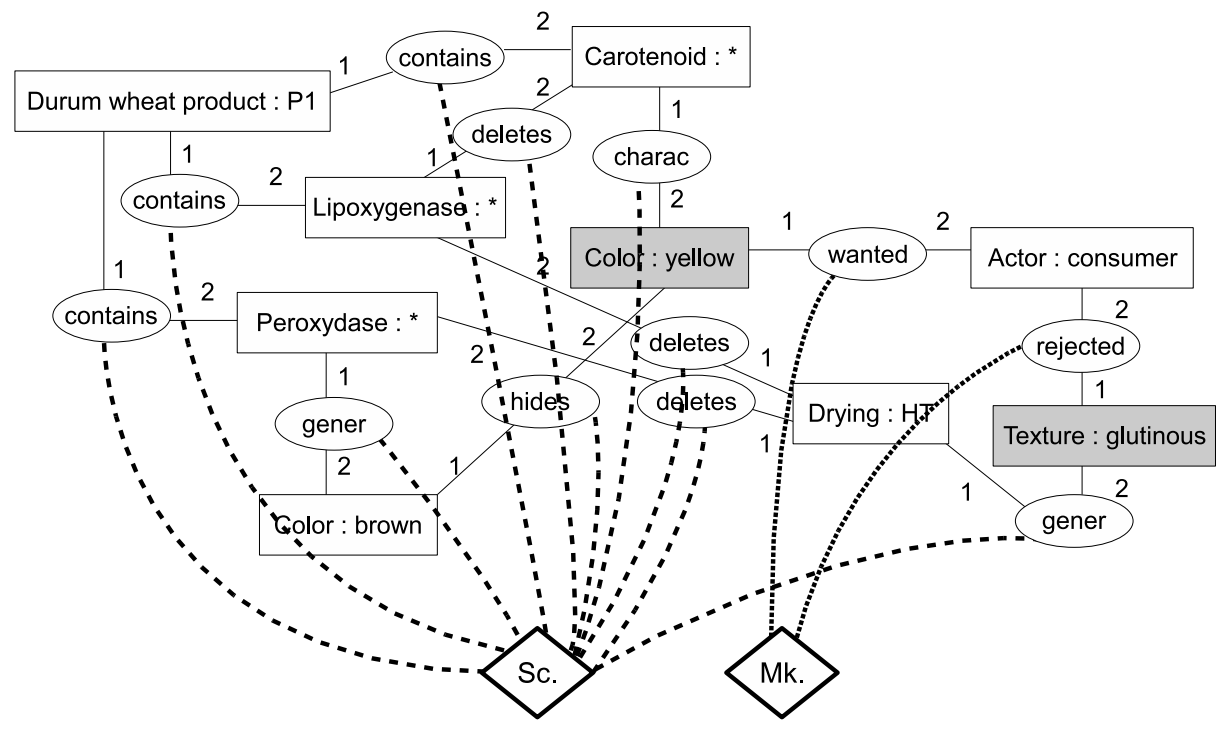

Figure 4. Answers to the query

\section{Formalism}

An ordered bipartite graph is a triple which consists of a set of concept nodes, a set of relation nodes and a set of mappings between the relation nodes and nonempty finite sequences over concept nodes. An ordered bipartite graph with just one relation node is called a star graph. We consider a special kind of subgraphs for our modelling purposes, namely spanned subgraphs. A spanned subgraph induced by a set of relation nodes consists of the set of relation nodes, the edges incident with these and the corresponding concept nodes.

\section{Definition 1. (Ordered Bipartite Graph)}

A triple $G=\left(V_{C}, V_{R}, N_{G}\right)$ is called an ordered bipartite graph if - $V_{C}$ and $V_{R}$ are finite disjoint sets, $\left(V_{G}:=V_{C} \cup V_{R}\right.$ is the vertices set of $\left.G\right)$, and

- $N_{G}: V_{R} \rightarrow V_{C}^{+}$is a mapping; $V_{C}^{+}$is the set of all finite nonempty sequences over $V_{C}$.

For $r \in V_{R}$ with $N_{G}(r)=c_{1} \ldots c_{k}, d_{G}(r):=k$ is the degree of $r$ in $G$ and $N_{G}^{i}(r):=c_{i}$ is the $i$-neighbour of $r$ in $G$. The set of (distinct) neighbours of $r$ is denoted $\bar{N}_{G}(r)$. 
The multiset $E_{G}$ of edges of $G$ is $E_{G}=\left(\{c, r\} \mid c \in V_{C}, r \in V_{R}\right.$ and $\exists i$ such that $\left.N_{G}^{i}(r)=c\right)$.

We further assume that for each $c \in V_{C}$ there is $r \in V_{R}$ and $i \in \mathbb{N}$ such that $c=N_{G}^{i}(r)$ ( $G$ has no isolated vertices $)$.

An ordered bipartite graph $G=\left(V_{C}, V_{R}, N_{G}\right)$ with $\left|V_{R}\right|=1$ is called a star graph.

If $G=\left(V_{C}, V_{R}, N_{G}\right)$ is an ordered bipartite graph and $A \subseteq V_{R}$, the subgraph spanned by $A$ in $G$ is the graph $\mathbf{G}[\mathbf{A}]:=\left(V_{C}^{1}, A, N_{G}^{1}\right)$, where $N_{G}^{1}$ is the restriction of $N_{G}$ to $A$ and $V_{C}^{1}=\left\{c \in V_{C} \mid \exists r \in A\right.$ and $\exists i \in \mathbb{N}$ such that $\left.c=N_{G}^{i}(r)\right\}$.

If $A=\{r\}$, then we simply write $G[r]$, which is referred to as the star subgraph spanned by $r$ in $G$. Clearly, the graph $G$ can be expressed as the union of its star subgraphs: $G=\cup_{r \in V_{R}} G[r]$.

Ordered bipartite graphs are appropriate tools to represent and visualize (directed) hypergraphs. Visually, an ordered bipartite graph $G=\left(V_{C}, V_{R}, N_{G}\right)$ can be represented using boxes for vertices in $V_{C}$, ovals for vertices in $V_{R}$ and integer labelled simple curves (edges) connecting boxes and ovals: if $c$ and $r$ are such that $c=N_{G}^{i}(r)$, then we have an edge with label $i$ connecting the box labelled $c$ to the oval labelled $r$ (the labels of the vertices are depicted outside of the corresponding shape, and are used as visual marks only if it is necessary to make the diagram more clear).

We also need some additional graph theoretical notations. If $D=(V, E)$ is a DAG (Directed Acyclic Graph), then a source (sink) in $D$ is any node $v$ of $D$ such that there is no entering (leaving) arc in (from) $v$.

A hypergraph is a pair $H=(V, \mathcal{P}(H))$, where $V$ is a nonempty finite set (the vertices set of $H$ ), and $\mathcal{P}(H)$ is a family of nonempty subsets of $V$. Each member $P$ of $\mathcal{P}(H)$ is a hyperedge of $H$.

The next two definitions, following the line of [1], introduce the concepts of support and Conceptual Graphs. A support is a structure that provides the background knowledge about the information to be represented in the Conceptual Graphs. It consists of a concept type hierarchy, a relation type hierarchy, a set of individual markers that refer to specific concepts and a generic marker, denoted by ${ }^{*}$, which refers to an unspecified concept.

Definition 2. (Support)

$A$ support is a 4-tuple $S=\left(T_{C}, T_{R}, \mathcal{I}, *\right)$ where:

- $T_{C}$ is a finite partially ordered set (poset), $\left(T_{C}, \leq\right)$, of concept types, defining a type hierarchy (specialization hierarchy: $\forall x, y \in T_{C} x \leq y$ means that $x$ is a subtype of $y$ ) and which has a greatest element $T_{C}$, the universal type.

- $T_{R}$ is a finite set of relation types partitioned into $k$ posets $\left(T_{R}^{i}, \leq\right)_{i=1, k}$ of relation types of arity $i(1 \leq i \leq k)$, where $k$ is the maximum arity of a relation type in $T_{R}$. Each $\left(T_{R}^{i}, \leq\right)_{i=1, k}$ has a greatest element, the universal type $T_{T_{R}^{i}}$.

- I is a countable set of individual markers, used to refer specific concepts.

- * is the generic marker used to refer to an unspecified concept (having, however, a specified type).

- The sets $T_{C}, T_{R}, \mathcal{I}$ and $\{*\}$ are mutually disjoint and $\mathcal{I} \cup\{*\}$ is partially ordered by $x \leq y$ iff $x=y$ or $y=*$. 
A Conceptual Graph is a structure that depicts factual information about the background knowledge contained in its support. This information is presented in a visual manner as an ordered bipartite graph, whose nodes have been labelled with elements from the support. The label $\lambda(v)$ is inserted in the shape representing the node $v$.

Definition 3. (Conceptual graph) A (simple) Conceptual Graph (CG) is a triple $S G=[S, G, \lambda]$, where:

- $S=\left(T_{C}, T_{R}, \mathcal{I}, *\right)$ is a support;

- $G=\left(V_{C}, V_{R}, N_{G}\right)$ is an ordered bipartite graph;

- $\lambda$ is a labelling of the vertices of $G$ with elements from the support $S: \forall r \in$ $V_{R}, \lambda(r) \in T_{R}^{d_{G}(r)} ; \forall c \in V_{C}, \lambda(c) \in T_{C} \times(\mathcal{I} \cup\{*\})$.

We introduce now the notion of a Conceptual Graph Assembly (CGA) as a structure which consists of a Conceptual Graph (CG) and a hypergraph on the $\mathrm{CG}$ 's relation nodes. Each hyperedge defines a $\mathrm{CG}$ subgraph which is a member of the CGA.

\section{Definition 4. (Conceptual Graph Assembly)}

Let $S=\left(T_{C}, T_{R}, \mathcal{I}, *\right)$ be a support, $S G=[S, G, \lambda]$ a Conceptual Graph without isolated concept vertices, and let $H=\left(V_{R}, \mathcal{P}(H)\right)$ be a hypergraph on the set $V_{R}$ of all relation vertices of $G$.

The pair $C G A=(S G, H)$ is called a Conceptual Graph Assembly. The members of $C G A=(S G, H)$ are the Conceptual Graphs $S G_{P}=\left(S, G[P], \lambda_{P}\right)$, where $P \in \mathcal{P}(H)$ is any hyperedge of $H, G[P]$ is the subgraph of $G$ spanned by the hyperedge $P$, and $\lambda_{P}$ is the restriction of $\lambda$ on the set of vertices of $G[P]$.

Note that any simple Conceptual Graph $S G$ (without isolated concept vertices) can be viewed as a Conceptual Graph Assembly with a single member, by taking $H$ as a hypergraph with a single hyperedge $\mathcal{P}(H)=\left(V_{R}\right)$, containing all relation vertices of $G$.

Each hyperedge can be considered as a given world; each $\mathrm{CG}$ member of a CGA thus provides information available in this world. The hypergraph $H$ can be given explicitly or implicitly, by a combinatorial property of its hyperedges. The latter case will be developed in Section 4 .

Explicitly, the hypergraph $H$ can be represented as a bipartite graph with one node class $V_{R}$, and the other (disjoint) class having a node $v_{P}$ for each hyperedge $P \in \mathcal{P}(H)$, connected by edges to the relation nodes belonging to $P$. In the visual representation of the Conceptual Graph $S G$, this new set of vertices, $V_{P}$, representing the members of the CGA, can be designated as diamonds. This was illustrated in the example of figure 2 .

From a knowledge representation point of view, this tripartite graph structure has the property that the information is well organized in order to facilitate reasoning (inferences) and, at the same time, presents itself as a visual medium of expression. 
CGs are provided with logical semantics; more precisely, an operator $\theta$ is considered, which assigns to every support $S$ a set of FOL formulas $\theta(S)$ and maps each simple Conceptual Graph $G$ to a conjunctive, existential closed FOL formula $\theta(G)$. $\theta$ can be the well-known Sowa's operator $\Phi$, or a variant of it $\Psi$ discussed in [3]. A logical semantics of CGAs can be defined as follows.

\section{Definition 5. (CGA logical semantics)}

Let $\theta$ be a logical semantics for $C G s$, and $C G A=(S G, H)$ a Conceptual Graph Assembly. Then, $\theta(C G A)$ is the disjunction of the formulas assigned by $\theta$ to the members of $C G A$, that is,

$$
\theta(C G A)=\vee_{P \in \mathcal{P}(H)} \theta\left(S G_{P}\right)
$$

For example, consider the CGA $C G A_{\text {grouping }}$ described in Figure 5 and $\theta=\Phi$. Then, $\Phi\left(C G A_{\text {grouping }}\right)=[$ Color $($ yellow $) \wedge$ Color $($ brown $) \wedge$ hides $($ brown, yellow $)] \vee$ $[$ Color $($ yellow $) \wedge$ Actor $($ consumer $) \wedge$ Texture $($ glutinous $) \wedge$ wanted $($ yellow, consumer $) \wedge$ rejected(glutinous, consumer)].

Projection [12] is the fundamental operation on simple Conceptual Graphs since it can be used to define a preorder on the set of CGs based on the same support. If $S G=\left(G, \lambda_{G}\right)$ and $S F=\left(F, \lambda_{F}\right)$ are two CG's defined on the same support $S$, then a projection from $S G$ to $S F$ is a mapping $\Pi: V_{C}(G) \cup V_{R}(G) \rightarrow$ $V_{C}(F) \cup V_{R}(F)$ such that

- $\Pi\left(V_{C}(G)\right) \subseteq V_{C}(F)$ and $\Pi\left(V_{R}(G)\right) \subseteq V_{R}(F)$;

- $\forall c \in V_{C}(G), \forall r \in V_{R}(G)$ if $c=N_{G}^{i}(r)$ then $\Pi(c)=N_{F}^{i}(\Pi(r))$

- $\forall v \in V_{C}(G) \cup V_{R}(G) \lambda_{G}(v) \geq \lambda_{F}(\Pi(v))$.

If there is a projection from $S G$ to $S F$ then $S G$ subsumes $S F$, which is denoted $S G \geq S F$. This subsumption relation is a preorder on the set of all CG's defined on the same support. Subsumption checking is an NP-complete problem [1].

The notion of projection can be extended to CGAs. In the next definition we consider three forms of projection (weak, mild and strong) under two scenarios: projecting a CGA to a CG or projecting a CGA to a CGA. Intuitively we need different projection mechanisms to account for the very nature of Conceptual Graph Assemblies: commensalism. More precisely when trying to project a Conceptual Graph Assembly in a simple conceptual graph we need to distinguish from the case when the information encoded in the simple conceptual graph is contained in at least one world, all of worlds, or the conceptual graph itself represented by the Conceptual Graph Assembly. Similarly when projecting two Conceptual Graph Assemblies (which is a generalization of the previous case) we have to consider the same three possible situations.

Definition 6. (CGA Projection)

I. Let $C G A^{1}=\left(S G^{1}, H^{1}\right)$ be a $C G A$ and $S G^{2}$ a $C G$. Then

- weak projection : $C G A^{1} \geq_{w} S G^{2}$ if there is $P^{1} \in \mathcal{P}\left(H^{1}\right)$ such that $S G_{P^{1}}^{1} \geq$ $S G^{2}$.

- mild projection : $C G A^{1} \geq_{m} S G^{2}$ if $S G_{P^{1}}^{1} \geq S G^{2}$ for each $P^{1} \in \mathcal{P}\left(H^{1}\right)$. 
- strong projection : $C G A^{1} \geq_{s} S G^{2}$ if $S G^{1} \geq S G^{2}$.

II. Let $C G A^{1}=\left(S G^{1}, H^{1}\right)$ and $C G A^{2}=\left(S G^{2}, H^{2}\right)$ be two $C G A s$. Then - weak projection : $C G A^{1} \geq_{w} C G A^{2}$ if there are $P^{1} \in \mathcal{P}\left(H^{1}\right)$ and $P^{2} \in$ $\mathcal{P}\left(H^{2}\right)$ such that $S G_{P^{1}}^{1} \geq S G_{P^{2}}^{2}$.

- mild projection : $C G A^{1} \geq_{m} C G A^{2}$ if for each $P^{1} \in \mathcal{P}\left(H^{1}\right)$ there is $P^{2} \in$ $\mathcal{P}\left(H^{2}\right)$ such that $S G_{P^{1}}^{1} \geq S G_{P^{2}}^{2}$.

- strong projection : $C G A^{1} \geq_{s} S G^{2}$ if there is a projection $\Pi$ from $S G^{1}$ to $S G^{2}$ such that the restriction of $\Pi$ to the relation vertices of $S G^{1}$ is a homomorphism from $\mathrm{H}^{1}$ to $\mathrm{H}^{2}$.

Note that in the case when $C G A^{1}=\left(S G^{1}, H^{1}\right)$ is a simple conceptual graph (i.e. it contains a single member), weak, mild and strong projection are identical.

The following theorem can be easily deduced from the above definitions and further explains the need for different combinatorial degrees of subsumption. Note that strong and mild projection can give extra information with regard to the associated CGA logical semantics. When defined from a CGA to a CG, weak projection preserves the soundness and completeness of CG projection; when defined on two CGAs, it only preserves the soundness.

Theorem 1. I. Let $C G A^{1}=\left(S G^{1}, H^{1}\right)$ be a $C G A$ and $S G^{2}$ a $S C G$. Then the following implications hold:

$$
C G A^{1} \geq_{s} S G^{2} \Rightarrow C G A^{1} \geq_{m} S G^{2} \Rightarrow C G A^{1} \geq_{w} S G^{2} .
$$

Furthermore, if $\theta$ is a logical semantics for $C G$ s such that $S G$ projection is sound and complete with respect to $\theta$ then

$$
C G A^{1} \geq_{w} S G^{2} \Leftrightarrow \theta(S), \theta\left(S G^{2}\right) \models \theta\left(C G A^{1}\right) .
$$

II. Let $C G A^{1}=\left(S G^{1}, H^{1}\right)$ and $C G A^{2}=\left(S G^{2}, H^{2}\right)$ be two CGAs. Then the following implications hold:

$$
C G A^{1} \geq_{s} C G A^{2} \Rightarrow C G A^{1} \geq_{m} C G A^{2} \Rightarrow C G A^{1} \geq_{w} C G A^{2} .
$$

If $\theta$ is a logical semantics for $C G$ s such that $S G$ projection is sound and complete with respect to $\theta$ then

$$
\begin{gathered}
C G A^{1} \geq_{w} C G A^{2} \Leftrightarrow \\
\text { there is } P^{2} \in \mathcal{P}\left(H^{2}\right) \text { s.t. } \theta(S), \theta\left(S G_{P^{2}}^{2}\right) \models \theta\left(C G A^{1}\right) .
\end{gathered}
$$

Proof:

Part I. Suppose that $C G A^{1} \geq_{s} S G^{2}$. It follows that $\Pi_{G^{1} \rightarrow G^{2}} \neq \emptyset$. Let $\pi \in$ $\Pi_{G^{1} \rightarrow G^{2}}$ and $P^{1} \in \mathcal{P}\left(H^{1}\right)$. $\pi_{1}$, the restriction of $\pi$ to the vertices of $\left[P^{1}\right]_{G^{1}}$, is a projection from $S G_{P^{1}}^{1}$ to $S G^{2}$. Therefore $S G_{P^{1}}^{1} \geq S G^{2}$ for each $P^{1} \in \mathcal{P}\left(H^{1}\right)$, that is $C G A^{1} \geq_{m} S G^{2}$. The implication $C G A^{1} \geq_{m} S G^{2} \Rightarrow C G A^{1} \geq_{w} S G^{2}$ is obvious by the definition of CGA projection. 
Let $\theta$ be a logical semantics for CGs such that SG projection is sound and complete with respect to $\theta$.

If $C G A^{1} \geq{ }_{w} S G^{2}$, then there is $P^{1} \in \mathcal{P}\left(H^{1}\right)$ such that $S G_{P^{1}}^{1} \geq S G^{2}$. By the soundness of $\theta$, we have $\theta(S), \theta\left(S G^{2}\right) \models \theta\left(S G_{P^{1}}^{1}\right)$. Now, by the definition of CGA semantics, $\theta\left(S G_{P^{1}}^{1}\right) \models \theta\left(C G A^{1}\right)$, and therefore $\theta(S), \theta\left(S G^{2}\right) \models \theta\left(C G A^{1}\right)$.

If $\theta(S), \theta\left(S G^{2}\right) \models \theta\left(C G A^{1}\right)$, it follows that there is a term in the disjunction $\theta\left(C G A^{1}\right)$, say $\theta\left(S G_{P^{1}}^{1}\right)$, where $P^{1} \in \mathcal{P}\left(H^{1}\right)$, such that $\theta(S), \theta\left(S G^{2}\right) \models \theta\left(S G_{P^{1}}^{1}\right)$. By the completeness of $\theta$, we obtain that $S G_{P^{1}}^{1} \geq S G^{2}$. We have obtained that there is $P^{1} \in \mathcal{P}\left(H^{1}\right)$ such that $S G_{P^{1}}^{1} \geq S G^{2}$ and, by the definition of CGA projection, $C G A^{1} \geq_{w} S G^{2}$ holds.

Part II. Suppose that $C G A^{1} \geq_{s} C G A^{2}$. It follows, by the definition of CGA projection, that there is $\pi$ a projection from $S G^{1}$ to $S G^{2}$ such that $P^{2}=\pi\left(P^{1}\right) \in$ $\mathcal{P}\left(H^{2}\right)$, for each $P^{1} \in \mathcal{P}\left(H^{1}\right)$. Obviously, $S G_{P^{1}}^{1} \geq S G_{P^{2}}^{2}$. Therefore, for each $P^{1} \in \mathcal{P}\left(H^{1}\right)$ there is $P^{2} \in \mathcal{P}\left(H^{2}\right)$ such that $S G_{P^{1}}^{1} \geq S G_{P^{2}}^{2}$, that is, $C G A^{1} \geq_{m}$ $C G A^{2}$

If $C G A^{1} \geq_{w} C G A^{2}$, then there is $P^{1} \in \mathcal{P}\left(H^{1}\right)$ and $P^{2} \in \mathcal{P}\left(H^{2}\right)$ such that $S G_{P^{1}}^{1} \geq S G_{P^{2}}^{2}$. By the soundness of $\theta$, we have $\theta(S), \theta\left(S G^{2}\right) \models \theta\left(S G_{P^{1}}^{1}\right)$. Now, by the definition of CGA logical semantics, $\theta\left(S G_{P^{1}}^{1}\right) \models \theta\left(C G A^{1}\right)$, and therefore $\theta(S), \theta\left(S G^{2}\right) \models \theta\left(C G A^{1}\right)$.

Conversely, if there is $P^{2} \in \mathcal{P}\left(H^{2}\right)$ s.t. $\theta(S), \theta\left(S G_{P^{2}}^{2}\right) \models \theta\left(C G A^{1}\right)$, then it follows that there is a term in the disjunction $\theta\left(C G A^{1}\right)$, say $\theta\left(S G_{P^{1}}^{1}\right)$, where $P^{1} \in$ $\mathcal{P}\left(H^{1}\right)$, such that $\theta(S), \theta\left(S G_{P^{2}}^{2}\right) \models \theta\left(S G_{P^{1}}^{1}\right)$. By the completeness of $\theta$, we obtain that $S G_{P^{1}}^{1} \geq S G_{P^{2}}^{2}$. Therefore, we have obtained that $C G A^{1} \geq{ }_{w} C G A^{2}$.

\section{Conceptual Modelling using CGAs}

As mentioned in the previous section, the hypergraph $H$ defined on the Conceptual Graph Assemblies relation nodes can be given explicitly (a list of the hyperedges of $H$ is provided) or implicitly. This section details the latter technique and shows how CGAs can be effectively used for knowledge modelling.

$H$ is given by specifying a property of its hyperedges. In this way, it is possible to represent, in a succinct manner, an exponential number of members in the CGA. However, if it is necessary, the explicit list of the hyperedges can be generated. Let us give some interesting ways to specify the above property.

(Di)graphs On the set $V_{R}$ of all relation vertices of the CG $G$, a graph (or digraph) $H G$ is provided. The edges (or directed edges) of the graph $H G$ express some links between their extremities. $\mathcal{P}(H)$ is described as a usual family of subsets of the vertices set of $H G$ having graph theoretical significance.

For example, if $D$ is an arbitrary DAG on the set $V_{R}$ of all relation vertices of $G$, we can take $\mathcal{P}(H)$ as the family of vertices of all paths in $D$ starting from a source and ending in a sink. The acyclicity condition assures that each path starting in a source must reach a sink. If it is necessary, a dummy source and a dummy sink are added in order to increase the visual quality of the digraphs 
considered (this fictive nodes are not considered when the hyperedges of $H$ are constructed).

Another example can be obtained if we consider a graph $H G$ on the set $V_{R}$ of all relation vertices of $G$ with the set of edges expressing a compatibility relation. For example, an edge $\left\{v_{r^{1}}, v_{r^{2}}\right\} \in E(H G)$ means that the facts expressed by $v_{r^{1}}$ and $v_{r^{2}}$ in the CG $G$ can be considered in the same time in order to describe a complex factual information. Taking $\mathcal{P}(H)$ as the family of vertices of all maximal (w.r.t. set inclusion) cliques in $H G$, we obtain a CGA with an exponential number of members, which could be an elegant and efficient representational mechanism.

Conceptual grouping Let $S=\left(T_{C}, T_{R}, \mathcal{I}, *\right)$ be a support, $S G=[S, G, \lambda]$ a Conceptual Graph without isolated concept vertices and $C G A^{1}=\left(S G, H^{1}\right)$ a CGA. Let $T H \subseteq T_{C}$ be a given set of threshold concept types and let $V_{T H} \subseteq V_{C}$ the set of all concept vertices $v_{c}$ of the graph $G$, with the property that if $\lambda\left(v_{c}\right)=$ type $\left._{v_{c}}, r e f_{v_{c}}\right)$ then $\exists t \in T H$ such that type $_{v_{c}} \geq t\left(V_{T H}\right.$ contains the vertices of $G$ designating objects having the type "above" the prescribed threshold $T H$ ).

Taking $\mathcal{P}(H)$ as the family of all maximal (w.r.t. inclusion) subsets $P$ of the members $P^{1}$ of $C G A^{1}$ such that $N_{G}(P) \subseteq V_{T H}$, we obtain a new CGA whose members describe only the facts about objects having a type above the threshold set $T H$ in the hierarchy given by $S$.

For example, starting from the CGA of figure 2, with $T H=\{$ Food product, Color, Texture, Person $\}$, we obtain the new CGA shown in figure 5. This CGA contains two members $P_{1}$ and $P_{2} . P_{1}$ indicates that the brown color hides the yellow one. $P_{2}$ indicates that the yellow color is wanted by the consumer and that the glutinous texture is rejected by the consumer. The interest of such a transformation can be, for instance, to determine parts of the knowledge base that can be easily understood by a wide public, due to the non-specific vocabulary used in the concepts.

Transversal methods Let $C G A^{1}=\left(S G, H^{1}\right)$ be a CGA. Taking $\mathcal{P}(H)$ as the family of all subsets $P$ of $V_{R}$ with the property that $P \cap P^{1} \neq \emptyset$, for each $P^{1} \in \mathcal{P}\left(H^{1}\right)$, we obtain a new CGA $C G A=(S G, H)$ with interesting combinatorial connections with the first one. For example, let us suppose that $C G A^{1}=\left(S G, H^{1}\right)$ satisfies the property that $\forall P^{1} \in \mathcal{P}\left(H^{1}\right)$ and $\forall P^{2} \in \mathcal{P}\left(H^{1}\right)$, if $P^{1} \subseteq P^{2}$ then $P^{1}=P^{2}$; if we take in $\mathcal{P}(H)$ only minimal transversal (that is minimal subsets, w.r.t. sets inclusion, of $V_{R}$ intersecting all members of $H^{1}$ ) then the hypergraph $H$ has the property that its minimal transversals are precisely the members of the initial hypergraph $H^{1}$.

Another example in this category comes from the integration framework described in [4]. Let $S G^{i}=\left[S^{i}, G^{i}, \lambda^{i}\right], i \in[0, n]$, be a set of $n+1$ CGs. For each relation node $r_{j}^{0} \in V_{R}^{0}=\left\{r_{1}^{0}, \ldots, r_{m}^{0}\right\}$, a set $\mathbb{R}\left(r_{j}^{0}\right)$ of triples is provided. Each such triple, $(i, A, w) \in \mathbb{R}\left(r_{j}^{0}\right)$ specifies a rewriting rule of $r_{j}^{0}$ in $S G^{i}: r_{j}^{0}$ is translated in the spanning subgraph $[A]_{G^{i}}$, and the $k=\operatorname{deg}_{G^{0}}\left(r_{j}^{0}\right)$ neighbors of $r_{j}^{0}$ in 


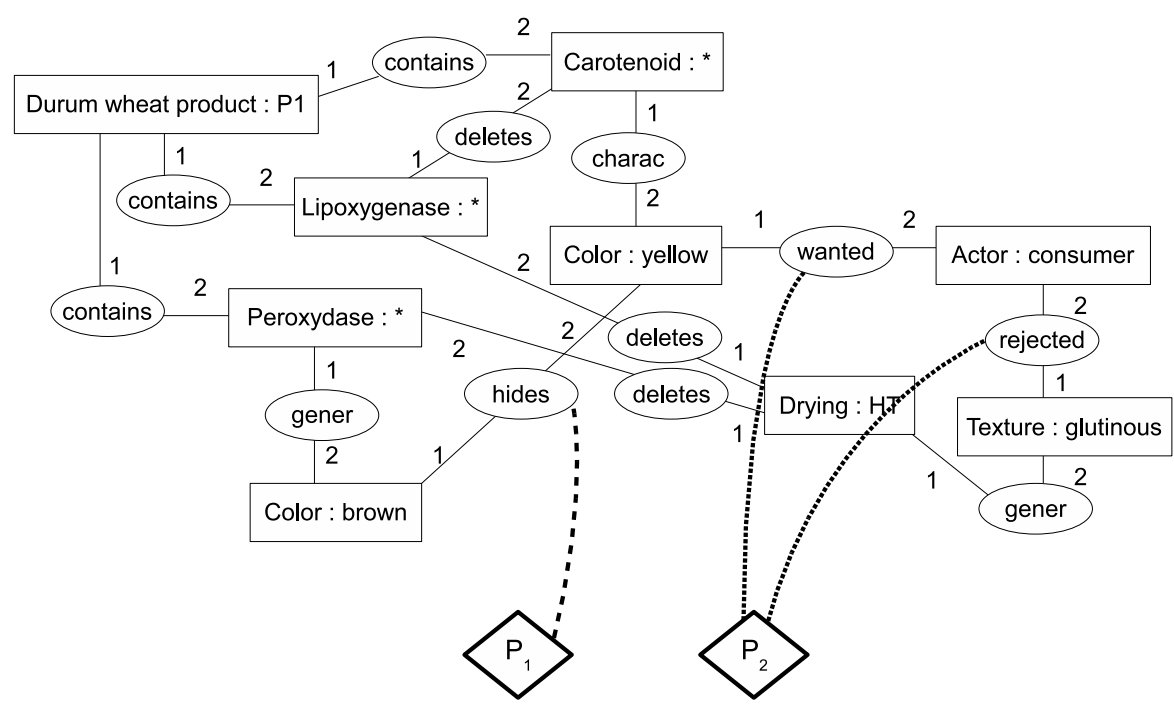

Figure 5. The new obtained CGA

$G^{0}$ are represented by the sequence $w=w^{1}, \ldots, w^{k}$ of concept nodes in $[A]_{G^{i}}$ : $N_{G^{0}}^{1}\left(r_{j}^{0}\right)$ is represented by $w^{1}, \ldots, N_{G^{0}}^{k}\left(r_{j}^{0}\right)$ is represented by $w^{k}$.

Now, for each transversal $T$ of the hypergraph $\mathbb{R}=\left(\mathbb{R}\left(r_{1}^{0}\right), \ldots, \mathbb{R}\left(r_{m}^{0}\right)\right)$, a hyperedge is added to the hypergraph $\mathcal{T}^{i}$ by considering the union of the relation nodes sets of $T$ contained in $V_{R}^{i}$. In this way, $n$ CGAs, $C G A^{i}=\left(S G^{i}, \mathcal{T}^{i}\right)$, are obtained.

Assisting reasoning Let $S G=\left[S, G, \lambda_{G}\right]$ and $S Q=\left[S, Q, \lambda_{Q}\right]$ be two CGs defined on the same support $S$ such that $S Q \geq S G$.

If $\Pi_{Q \rightarrow G}=\{\pi \mid \pi$ is a projection from $S Q$ to $S G\}$, then we can consider $\operatorname{Occ}(Q, G)=\left(\pi\left(V_{R}^{Q}\right) \mid \pi \in \Pi_{Q \rightarrow G}\right)$. Taking $H=\left(V_{R}^{G}, O c c(Q, G)\right)$ we obtain a CGA $C G A=(S G, H)$ which gives all the occurences of the query $S Q$ in $S G$. For some usual query $S Q$ this CGA can be pre-computed in order to have fast response time. With the same goal of efficiency, the following CGA can be considered.

Let $S G=[S, G, \lambda]$ be a Conceptual Graph and $\mathcal{M}$ a model for the support $S=\left(T_{C}, T_{R}, \mathcal{I}, *\right)$. Suppose that $\mathcal{M} \not \models S G$ and let us consider the CGA $C G A=$ $(S G, H), H=\left(V_{R}^{G}, \mathcal{P}(H)\right)$, where $\mathcal{P}(H)=\left(I \mid I \subset V_{R}^{G}\right.$ and $\left.\mathcal{M} \models[I]_{G}\right)$. It is easy 
to see that $H$ is an independence system on $V_{R}^{G}$, that is, if $I \in \mathcal{P}(H)$ and $I_{1} \subseteq I$, then $I_{1} \in \mathcal{P}(H)$.

\section{Conclusions}

In this paper we proposed a semantically sound syntactic extension to Conceptual Graphs: Conceptual Graph Assemblies (CGAs), and defined several reasoning mechanisms, based on the projection operation. We showed that CGAs provide increased representational power. We proposed several modelling scenarios and illustrated through an example in the agri-food domain the applicability of this extension in practice, in particular for the representation of multiple viewpoints on the same situation.

Conceptual Graph Assemblies are a flexible, versatile way of representing interrelated facts, concurrent events or possible scenarios. In future work we plan to explore two directions of modelling with CGAs: modelling temporal information, by attaching a temporal value to the relation nodes of the conceptual graph prior to defining the CGA by the means of this "stamp" value; modelling multi-viewpoints reasoning such as conflict detection - that can be viewed e.g. as the projection of a negative constraint in the conceptual graph represented by a CGA -, and resolution proposals through argumentation and decision methods. We believe these are promising directions of work which will further demonstrate CGAs applicability.

\section{References}

1. M. Chein and M.-L. Mugnier. Conceptual graphs: Fundamental notions. Revue d'Intelligence Artificielle, 6(4):365-406, 1992.

2. M. Chein and M.-L. Mugnier. Positive nested conceptual graphs. In Proc. of the 5th Int'l Conf. on Conceptual Structures (ICCS'97), pages 95-109, 1997.

3. M. Chein, M.-L. Mugnier, and G. Simonet. Nested graphs: A graph-based knowledge representation model with FOL semantics. In Proc. of the 6th Int'l Conf. on the Principles of Knowl. Repres. and Reasoning (KR'98), pages 524-535. Morgan Kaufmann, 1998.

4. M. Croitoru and E. Compatangelo. Hierarchical knowledge-oriented specification for information integration. In Research and Development in Intelligent Systems XXII - Proc. of the 25th Int'l Conf. of the Brit. Comp. Soc. Specialist Group on Artif. Intell. (AI'2005), pages 60-73. Springer, 2005.

5. M. Croitoru and E. Compatangelo. Conceptual graph assemblies. In P. Hitzler, H. Scharfe, and P. Ohrstrom, editors, Contributions to ICCS 2006, 14 th International Conference on Conceptual Structures, pages 15-28. Aalborg University Press, 2006.

6. M. Croitoru and E. Compatangelo. Extending conceptual graphs for representing partial knowledge. In the 7th IJCAI International Workshop on Nonmonotonic Reasoning, Action and Change, 2007.

7. J. Esch. Contexts and Concepts, Abstraction Duals. In Proc. of the 2nd Int'l Conf. on Conceptual Structures (ICCS'94), pages 175-184, 1994. 
8. G. W. Mineau and O. Gerbé. Contexts: A Formal Definition of Worlds of Assertions. In Proc. of the 5th Int'l Conf. on Conceptual Structures (ICCS'97), pages 80-94, 1997.

9. A. Puder et al. Service Trading Using Conceptual Structures. In 3rd International Conference on Conceptual Structures, pages 59-73. University of California, Springer-Verlag, 1995.

10. M. Ribière and R. Dieng-Kuntz. A Viewpoint Model for Cooperative Building of an Ontology. In Proc. of the 10th Int'l Conf. on Conceptual Structures (ICCS'2002), pages 220-234, 2002.

11. J. Sowa. Knowledge Representation: Logical, Philosophical, and Computational Foundations. Brooks Cole Publishing Co., 2000.

12. J. F. Sowa. Conceptual Structures: Information Processing in Mind and Machine. Addison-Wesley, 1984.

13. R. Thomopoulos, M. Mugnier, and M. Leclère. Mapping contexts to vocabularies to represent intentions. In ECAI'06 Workshop on Contexts and Ontologies: Theory, Practice and Applications (co-2006), pages 44-46, 2006. 